\title{
DATOS DEMOGRÁFICOS DE ANIMALES DE COMPAÑÍA ATENDIDOS EN CONSULTA DE OFTALMOLOGÍA Y DE LAS PERSONAS A SU CARGO
}

\section{Demographic Data of Companion Animals Treated in an Ophthalmology Cuinic and of their Caretakers}

\author{
Beatriz Hugues H. ${ }^{1,3}$, Miguel Torres L. ${ }^{2}$
}

\section{Resumen}

\begin{abstract}
Se presenta una descripción de datos demográficos de animales de compañía ( $\mathrm{n}=610)$ que fueron asistidos en una consulta de oftalmología veterinaria en La Habana, Cuba, en 2012, así como de las personas a su cargo (n=529). Los datos fueron obtenidos de las historias clínicas. Los perros constituyeron la especie de mayor frecuencia (68\%), seguida del gato (22\%), hámster (5\%) y tortugas (4\%). Los perros fueron mayormente hembras (62\%) con edades de 1 a 5 años (35\%), donde los grupos raciales más frecuentes fueron los mestizos (37\%), el Pequinés (27\%) y el Cocker Spaniel (11\%). Las personas a cargo de las mascotas fueron mayormente mujeres (68\%), de 31 a 50 años de edad (55\%), con nivel medio o superior de instrucción (90\%), con vínculos laborales (68\%), que viven en compañía de otros familiares (92\%) y la vivienda se encuentra usualmente en los distritos cercanos al centro asistencial. El principal motivo de la tenencia de mascotas, especialmente de perros y gatos, fue por afecto ( 85 y $60 \%$, respectivamente).
\end{abstract}

Palabras clave: animales de compañía, responsables de mascotas, demografía

\section{Abstract}

Demographic data related to companion animals $(n=610)$ assisted in the ophthalmology veterinary clinic in Havana during 2012 and of their caretakers $(n=529)$ is described. Retrospective data were obtained from clinical records. The dogs were the most frequent species $(68 \%)$, followed by cats $(22 \%)$, hamster $(5 \%)$ and turtles $(4 \%)$. Dogs were mainly female (62\%), between 1 to 5 years old (35\%), and the most representative

\footnotetext{
${ }^{1}$ Instituto Nacional de Endocrinología, La Habana, Cuba

${ }^{2}$ Universidad Agraria de La Habana, Mayabeque, Cuba

${ }^{3}$ E-mail: bettymig@infomed.sld.cu
}

Recibido: 5 de diciembre de 2013

Aceptado para publicación: 4 de marzo de 2014 
breeds were undefined crossbreds (37\%), Pekingese (27\%) and Cocker Spaniel (11\%). The caretakers were mostly women $(68 \%)$ between 31 to 50 years old $(55 \%)$, with high school level of education (90\%), workers (68\%), living with other family members $(92 \%)$ and the house was usually in districts close to the clinic. The main reason for keeping pets, especially dogs and cats was affection (85 and $60 \%$ respectively).

Key words: companion animals, pet caretaker, demography

\section{INTRODUCCIÓN}

Los animales de compañía (las llamadas mascotas) son animales que se conservan con el propósito de brindar compañía o para el disfrute o entretenimiento de los dueños. En este sentido, perros y gatos son considerados animales afectivos por el vínculo estrecho que se establece entre estos y los seres humanos. Las aves y los peces pertenecen al grupo de ornamento, y los roedores, las tortugas pequeñas y los animales exóticos han llegado a constituir, para muchos, un miembro más de la familia (Damron, 2000; Mills, 2009).

La convivencia entre animales de compañía y seres humanos brinda beneficios para unos y otros. Los animales reciben cobijo, se les prodiga cariño y afecto, y reciben atención médica, lo cual repercute de forma favorable en su bienestar. Al habitar en las casas, decrece el número de animales que vagabundean por las calles y disminuye la posibilidad de transmisión de zoonosis y del deterioro ambiental (Merck, 2000; Martínez, 2008).

Diversos estudios avalan los efectos positivos que reciben los seres humanos al convivir con animales de compañía, especialmente en relación a la salud física (Allen et al., 2001; Millhouse-Flourie, 2004; Wood et al., 2005), mental (McConnell et al., 2011) y el control metabólico (Gutiérrez et al., 2007).
En las últimas décadas ha habido una tendencia creciente en la población cubana hacia la convivencia con animales de compañía, lo cual se manifiesta en el incremento de la atención asistencial en las clínicas y consultorios veterinarios (W. Hernández, La Habana, comunicación personal). Sin embargo, existe poca información sobre las características de los grupos humanos y de los animales de compañía que están en posesión de sus cuidadores. El conocimiento de estas características resulta importante en el marco de la epidemiología y la salud pública, entre otras, por la estrecha relación y vínculo entre seres humanos y animales.

Sobre estas bases se llevó a cabo el presente estudio para describir algunos datos demográficos, tanto de los animales que son atendidos en consultas veterinarias, como de las propias personas a cargo de ellos, utilizando como fuente de información los registros de consulta oftalmológica de la principal clínica veterinaria de La Habana.

\section{Materiales y Métodod}

Se realizó un estudio demográfico descriptivo cuantitativo a partir de los datos obtenidos de las historias clínicas de los animales de compañía que fueron atendidos en el año 2012 en la consulta de oftalmología de la clínica veterinaria «José Luis Callejas», situada en La Habana, Cuba. Se consideró la especie, edad, sexo y raza o línea, quedando 
Cuadro 1. Distribución porcentual por edad de las mascotas atendidas como pacientes en una clínica oftalmológica de La Habana, Cuba (2012)

\begin{tabular}{llc}
\hline Especie & Edad & $\begin{array}{c}\text { Frecuencia } \\
(\%)\end{array}$ \\
\hline Perro & $0-2$ meses & 20 \\
$(\mathrm{n}=417)$ & $3-11$ meses & 30 \\
& $1-5$ años & 35 \\
& $>6$ años & 15 \\
Gato & $0-2$ meses & 23 \\
$(\mathrm{n}=135)$ & $3-11$ meses & 27 \\
& $1-5$ años & 41 \\
& $>6$ años & 10 \\
Hámster & $<1$ año & 66 \\
$(\mathrm{n}=32)$ & $>1$ año & 34 \\
Tortuga & $1-4$ años & 100 \\
$(\mathrm{n}=26)$ & & \\
\hline
\end{tabular}

Cuadro 2. Distribución porcentual por razas de perros y gatos atendidos como pacientes en una clínica oftalmológica de La Habana, Cuba (2012)

\begin{tabular}{llc}
\hline Especie & Raza & $\begin{array}{c}\text { Frecuencia } \\
(\%)\end{array}$ \\
\hline Perro & Mestizo & 37 \\
$(\mathrm{n}=417)$ & Pekinés & 27 \\
& Cocker Spaniel & 11 \\
& Maltés & 9 \\
& Teckel & 9 \\
& Otros & 7 \\
Gato & Pelicorto americano & 95 \\
$(\mathrm{n}=135)$ & Siamés & 3 \\
& Otros & 2 \\
\hline
\end{tabular}

la muestra integrada por 417 perros, 135 gatos, 32 hámsteres (Mesocricetus auratus) y 26 jicoteas o tortugas pequeñas (Trachemys decusata $)($ total $=610)$.
En la recolección de información se incluyó, además, a las personas responsables de la tenencia de los animales $(\mathrm{n}=529)$, registrando sus nombres, edad, género, categoría ocupacional, nivel de escolaridad, municipio de residencia, tipo de convivencia (solo o con más personas en el hogar), así como su preferencia por determinado tipo de animal.

Los datos fueron sometidos a estadística descriptiva.

\section{Resultados y Discusión}

\section{Los Animales de Compañía}

Con base a la muestra en estudio, se pudo determinar una mayor tenencia de perros $(68 \%)$ y gatos $(22 \%)$, seguidos por otras especies (hámsteres: $5 \%$; tortugas: $4 \%$ ). Esta tendencia fue encontrada en forma similar en otros estudios realizados en Cuba (Beovides et al., 2011; Domínguez et al., 2011). Asimismo, estudios realizados en Perú (Alva et al., 2010; Arévalo et al., 2010) y Chile (Abarca et al., 2011), mostraron una mayor proporción de perros y gatos como mascotas, seguidos por aves ornamentales, roedores, tortugas y animales exóticos (chimpancés y zorros).

En el Cuadro 1 se muestra la distribución porcentual por edad de las mascotas mantenidas como tales; asimismo, en el Cuadro 2 se muestra la distribución porcentual de perros y gatos según la raza. La frecuencia de hembras y machos fue de 60 y $40 \%$ en perros, 62 y $38 \%$ en gatos, 56 y $44 \%$ en hámsteres y 50 y $50 \%$ en tortugas.

En la especie canina, el grupo etario de 1 a 5 años fue el más frecuente (35\%), así como los grupos raciales más representados fueron los mestizos (37\%) y pekineses (27\%). Asimismo, la proporción de hembras (62\%) fue notablemente superior a la de machos (Cuadros 1 y 2). En un estudio previo en la zona, Hugues et al. (2007d) hallaron distri- 
Cuadro 3. Distribución porcentual por edad y género de los dueños o tenedores de las mascotas $(n=529)$ atendidas como pacientes en una clínica oftalmológica de La Habana, Cuba (2012)

\begin{tabular}{llc}
\hline Variable & & $\begin{array}{c}\text { Frecuencia } \\
(\%)\end{array}$ \\
\hline Edad & $21-30$ & 16 \\
& $31-40$ & 30 \\
& $41-50$ & 25 \\
& $61-60$ & 14 \\
& $>60$ & 15 \\
Género & Femenino & 68 \\
& Masculino & 32 \\
\hline
\end{tabular}

buciones similares, aunque el grupo etario más frecuente estuvo compuesto por canes mayores de 5 años. Guerra et al. (2011) caracterizaron la población canina en la ciudad de Camagüey, Cuba, encontrando una mayor frecuencia de hembras (59\%) y de mestizos (60\%), pero los perros Cocker Spaniel y Pekinés se encontraban en tercer $(6 \%)$ y séptimo lugar $(2.7 \%)$. No obstante, en el estudio llevado a cabo por Morales et al. (2009) en Chile se encontró una mayor proporción de machos $(62 \%)$ que de hembras.

En el caso de los gatos, la raza Pelicorto Americano fue la raza predominante $(95 \%)$, característica que se ha mantenido desde el estudio realizado por Hugues et al. (2007a); sin embargo, la edad más común en el presente estudio fue de 1 a 5 años, en tanto que en el otro estudio fue de animales menores de un año. En referencia al sexo, el $62 \%$ de hembras fue similar al $64 \%$ de hembras en la ciudad de Camagüey (Domínguez et al., 2011), pero superior al 50\% registrado por Hugues et al. (2007a) en La Habana.
La distribución porcentual por edad (Cuadro 1) y sexo de los hámsteres fue similar a los resultados hallados por Hugues $e t$ al. (2007b) al estudiar la población de estos animales atendidos en consulta de oftalmología entre 1999 y 2006.

Las tortugas (jicoteas) que mayormente se registraron en la clínica fueron de la especie Trachemys decusata (Hugues et al., 2007c). En La Habana existe una clínica especializada en la atención de aves ornamentales, por lo cual no aparecen datos de esos animales en el presente estudio. No obstante, se sabe que las especies que son atendidas con mayor frecuencia son las cotorras, cateyes, canarios, cacatillos, periquitos y agapornis (I. Acosta, La Habana, comunicación personal).

\section{Los Dueños o Tenedores}

Los registros señalan una mayor frecuencia porcentual de mujeres $(68 \%)$ entre 31 y 50 años de edad (55\%) (Cuadro 3), donde el $68 \%$ trabajaba, el 20\% era ama de casa o jubilado y el restante $12 \%$ era estudiante.

El 90\% de las personas había alcanzado un nivel de escolaridad medio o superior (Fig. 1), en tanto que los municipios con mayor frecuencia de procedencia fueron aquellos más cercanos a la clínica (Fig. 1).

En el estudio de Beovides et al. (2011), donde se evaluó el cuidado de los animales de compañía en la ciudad de Santa Clara, se encontró una mayor frecuencia de hombres (53\%), con edades de 18 a 30 años (43\%), y con un nivel de escolaridad medio (59\%), los cuales son medianamente diferentes a los del presente estudio, especialmente en lo referente al género.

El 92\% de las personas manifestó que vivía en compañía de familiares, en tanto que el $8 \%$ tenía como única compañía a las mascotas. Asimismo, en el caso de perros, el $85 \%$ de las personas los poseía por tener un 

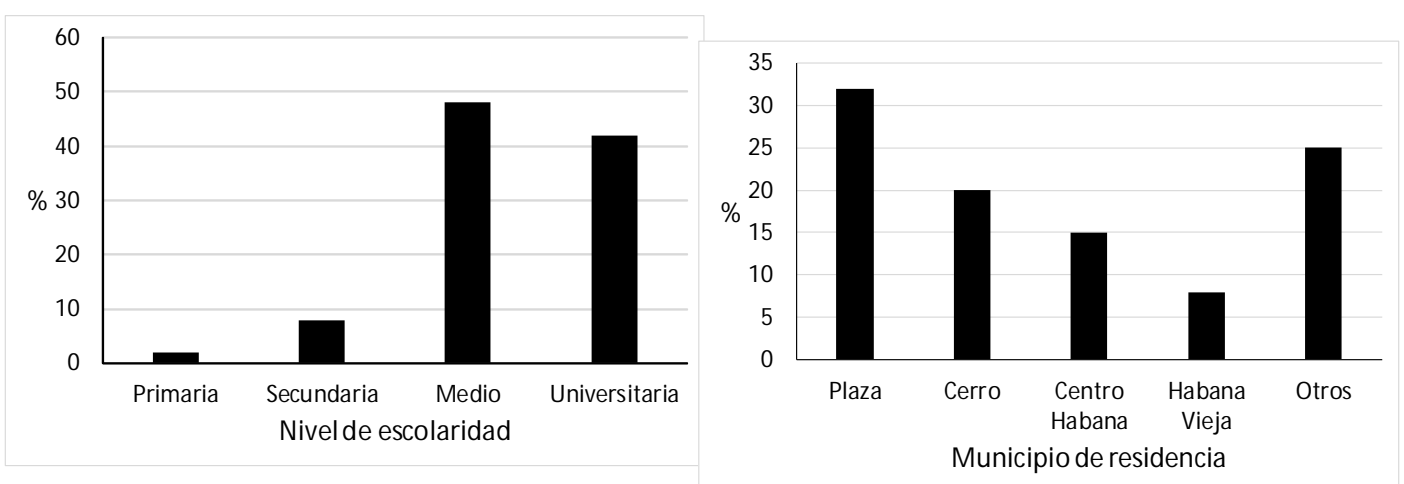

Figura 1. Distribución porcentual de dueños o tenedores de mascotas según su nivel de escolaridad (panel izquierdo) y del municipio de residencia (panel derecho).

vínculo afectivo con ellos, el $8 \%$ por motivos de caza (roedores principalmente) y el $7 \%$ por seguridad (cuidar la casa). En el caso de los gatos, el $60 \%$ de las personas los tenía por razones afectivas, el $25 \%$ afirmó que la función era para la caza de ratas y ratones, y el $15 \%$ alegó que los tenían tanto por el afecto que sentían hacia ellos como para el control biológico en la erradicación de roedores.

El motivo de la tenencia de animales es muy variable según el área geográfica. Por ejemplo, Ibarra et al. (2010) encontraron, al analizar la población de perros en Calera de Tango, Chile, que en el área urbana predominaba la razón de afectividad, mientras que en el área rural era por afectividad y seguridad (guardianía). Asimismo, Degregorio y Sommerfelt (2002) mostraron en Buenos Aires, Argentina, que la tenencia de animales de compañía era debida a razones dadas por el gusto personal (afecto) y seguridad. Por otro lado, Domínguez et al. (2011), en un estudio sobre las características de la tenencia de la población felina en la ciudad de Matanzas, Cuba, hallaron que la razón principal de la tenencia de gatos era de índole afectiva (52\%).

\section{Conclusiones}

- Los animales de compañía que son asistidos con mayor frecuencia en la consulta oftalmológica son los perros y los gatos, y dentro de estos, los adultos jóvenes y sobre todo las hembras.

- Las personas responsables de las mascotas llevadas a la consulta son mayormente mujeres jóvenes, de educación media y superior, con vínculos laborales, que poseen a estos animales principalmente por afectividad.

\section{Literatura Citada}

1. Allen K, Skykoff BE, Izzo JL.2001. Pet ownership, but not ACE inhibitor therapy, blunts homeblood pressure responses to mental stress. Hypertension 38:815-820.

2. Abarca K, López J, Peña A, López C. 2011. Tenencia y estado de salud de mascotas de niños inmunocomprometidos, con énfasis en enfermedades zoonóticas. Rev Chil Infect 28: 205-210. 
3. Alva R, Revilla E, Isla C. 2010. Convivencia con animales de compañía en niños y jóvenes estudiantes del distrito de Chiclayo, provincia de Chiclayo, Lambayeque- Perú. En: XXII Congreso Panamericano de Ciencias Veterinarias. Lima, Perú.

4. Arévalo W, Rachumi V, Eslava J. 2010. Tenencia responsable de animales de compañía en niños y jóvenes estudiantes del distrito de Ferreñafe, Lambayeque-Perú. En: XXII Congreso Panamericano de Ciencias Veterinarias. Lima, Perú.

5. Beovides LM, Silveira PE, Norman MO. 2011. Evaluación del cuidado de la salud de mascotas en el municipio Santa Clara, Cuba. En: VII Congreso Internacional de Ciencias Veterinarias. La Habana, Cuba.

6. Damron WS. 2000. Pet and companion animals. En: Introduction to animal science: Global, biological, social and industry perspectives. USA: Prentice Hall. p 569-579.

7. Degregorio OJ, Sommerfelt IE. 2002. Percepciones que tienen los médicos veterinarios en relación con la tenencia de animales en la Ciudad de Buenos Aires. Rev Med Vet 88: 144-148.

8. Domínguez H, Pérez Y, Barrios V. 2011 Características de la tenencia de la población felina en la ciudad de Matanzas. En: Vll Congreso Internacional de Ciencias Veterinarias. La Habana, Cuba.

9. Guerra Y, Echagarrúa Y, Marín E, Mencho JD, Marín A, Pascual T, et al. 2007. Factores que conllevan al abandono de perros en una región de Cuba. REDVET 8(12) [Internet]. Disponible en http://www.veterinaria.org/ revistas/redvet/n121207/120704.pdf

10. Gutiérrez G, Granados DR, Piar N. 2007. Interacciones humano-animal: características e implicaciones para el bienestar de los humanos. Rev Colomb Psicol 16: $163-183$
11. Hugues B, Navaroli F, Torres $M$. 2007a. Oftalmopatías comunes en gatos domésticos. En: Agrociencias 2007. Congreso Internacional de Ciencias Agropecuarias. La Habana, Cuba.

12. Hugues B, Navaroli F, Torres $M$. 2007b. Oftalmopatías comunes en hámsteres domésticos. En: Agrociencias 2007. Congreso Internacional de Ciencias Agropecuarias. La Habana, Cuba.

13. Hugues B, Navaroli $F$, Torres $M$. 2007c. Oftalmopatías comunes en jicoteas domésticas. En: Agrociencias 2007. Congreso Internacional de Ciencias Agropecuarias. La Habana, Cuba.

14. Hugues B, Navaroli $F$, Torres $M$. 2007d. Oftalmopatías comunes en perros domésticos. En: Agrociencias 2007. Congreso Internacional de Ciencias Agropecuarias. La Habana, Cuba.

15. Ibarra L, Makarena C, Illanes J. 2010. Comparación de las características demográficas de la oblación de perros en el área rural y urbana, calera de Tango, región metropolitana, Chile. En: XXII Congreso Panamericano de Ciencias Veterinarias. Lima, Perú.

16. McConnellAR, Brown CM, Shoda TM, Stayton LE, Martin CE. 2011. Friends with benefits: on the positive consequences of pet ownership. J Pers Soc Psychol 101: 1239-1252.

17. Martínez A. 2008. La terapia asistida por animales: una nueva perspectiva y línea de investigación en la atención a la diversidad. Indivisa Bol Estud Invest 9: $117-144$.

18. Merck. 2000. Comportamiento de los animales. Relación hombre animal. En: Manual Merck de Medicina Veterinaria. España: Ed Oceáno Centrum. p 345-367.

19. Millhouse-Flourie TJ. 2004. Physical, occupational, respiratory, speech, equine and pet therapies for mitochondrial disease. Mitochondrion 4: 549-558.

20. Mills DS. 2009. Dogs in society can prevent society going to the dogs. Vet $\mathrm{J}$ 179: 322-323. 
21. Morales MA, Varas C, Ibarra L. 2009. Caracterización demográfica de la población de perros de Viña del Mar, Chile. Arch Med Vet 41: 89-95.
22. Wood L, Giles-Corti B, Bulsara M. 2005. The pet connection: pets as a conduit for social capital? Soc Sci Med 61: 1159-1173. 